\title{
“NO DEJES QUE TE HECHICEN, ERES SABI@”. PROPUESTA PARA LA SOCIALIZACIÓN PREVENTIVA DE LA VIOLENCIA
}

\author{
"Don't let them bewitch you, you are wise". Proposal for the preventive social- \\ ization of violence
}

Isabel Vidaller Ferró

Fecha de recepción: 12/09/2018

Fecha de aceptación: 21/12/2018

RESUMEN: Presentamos una estrategia lúdica, enmarcada como una acción más dentro de un proyecto educativo que tiene como prioridad acabar con las situaciones de abuso y maltrato que se dan en nuestra sociedad y que se manifiestan en el entorno escolar. Se trata de una dinámica que ayuda a detectar información importante para enriquecer las situaciones de diálogo necesarias en la prevención y solución de conflictos. Hemos identificado y analizado las conductas irrespetuosas más frecuentes que generan sufrimiento y malestar en nuestro contexto escolar. Las conductas analizadas, las hemos tipificado en 4 tipos, creando para cada una de ellas un personaje que representa un pensamiento erróneo del que surgen sentimientos que no puede controlar y que marcan su conducta. Con las aportaciones del alumnado hemos definido el perfil de cada personaje, concretando el pensamiento clave de su error y el antídoto que lo contrarresta, es decir, una señal que puede evitar continuar por el camino equivocado y facilitar el rescate hacia una respuesta más sabia. Para poder difundir esta información de una manera asequible a todos los niños y niñas del centro y que fácilmente pudiera ser memorizada para poder ponerla en práctica en su vida real, hemos ideado un juego del tipo pilla-pilla que llamamos "no dejes que te hechicen, eres sabi@“" Con el desarrollo de esta dinámica estamos comprobando que tomar conciencia del pensamiento equivocado que está detrás de las conductas irrespetuosas ayuda progresivamente a desactivarlas, encontrando más fácilmente respuestas alternativas y por tanto un mayor margen de libertad en las acciones. Observamos también que la utilización de personajes facilita mucho el reconocimiento de los propios errores, gracias a la distancia simbólica que se crea entre la persona y su conducta. Como consecuencia aumenta el deseo y el compromiso para cambiar. Los personajes son una máscara que se puede poner y quitar, con ello se logra salir más fácilmente del encasillamiento o identificación de la persona con el rol de agresor o víctima. Y por último, creemos fundamental y prioritario para la acción educativa preservar y fortalecer la confianza básica de cada persona en su propio valor, es decir, lograr una buena autoestima, para conseguir interacciones donde la comunicación sea verdaderamente respetuosa.

PALABRAS CLAVE: Solución de conflictos, Autoestima, Juego, Aprendizaje social.

ABSTRACT: We present a playful strategy framed inside a broader educational project which main priority is to end the situations of social abuse manifested in the school environment. It is a dynamic that helps to detect information that is important to enrich the dialogue situations that are needed in the prevention and resolution of conflicts. We have identified and analyzed the most frequent disrespectful behaviors that generate suffering and discomfort in our school context. We have classified these conducts in 4 types, creating a character for each of them. Each character represents an erroneous thought from which uncontrollable feelings arise marking the different behaviors. With our students' contributions we have defined each character's profile, specifying the key thought that led to their erroneous attitude and the antidote that counteracts it; in other words, the signal that can facilitate the rescue towards a wiser response. To be able to disseminate this information to all the students in an accessible way making it easier to memorize so it can be applied in their real life conditions, 
we have designed a play tag-like game that we have called "don't let them bewitch you, you are wise". With the development of this dynamic we are proving that becoming aware of the wrong thinking that is behind disrespectful behaviors, helps progressively to deactivate them, finding alternative responses more easily and therefore providing a greater margin of freedom of action. We have also noticed that the use of characters greatly facilitates recognizing one's own mistakes, thanks to the symbolic distance that is created between the person and his/her behavior. As a result, the desire and commitment to change increases. The characters are a mask that can be put on and take off, thus breaking more easily the stereotype or identification with the role of aggressor or victim. And finally, we believe that is fundamental and a matter of priority for educational action to preserve and strengthen the basic confidence of each person in his/her own value, that is to say, achieve good self-esteem, to accomplish interactions where communication is truly respectful.

KEY WORDS: Conflict resolution, Self-esteem, Game, Social learning

\section{Introducción}

El patriarcado es la organización social dominante en la mayor parte del planeta, con un sistema de valores que está detrás del sufrimiento del ser humano desde hace varios miles de años hasta la actualidad. Se trata de una construcción social histórica obsoleta, que no es la única posible, de la que tenemos que salir para encontrar un modelo de funcionamiento coherente con la justicia que anhelamos, expresada en las declaraciones de los Derechos Humanos, donde el respeto a uno mismo, a los demás y a todo lo que nos rodea sean progresivamente una realidad.

Enmarcamos nuestra estrategia como una pequeña acción dentro de un proyecto educativo ambicioso que tiene como prioridad acabar con las situaciones de abuso y maltrato que se dan en nuestra sociedad y que se manifiestan en el entorno escolar, trabajando día a día para establecer un nuevo modelo de relaciones respetuosas donde el maltrato se detecte y erradique desde el primer momento.

Claudio Naranjo habla de una "mente patriarcal" que subyace al problema patriarcal de la sociedad, caracterizada como una sociedad en que las relaciones de dominio-sumisión interfieren en la capacidad de establecer vínculos solidarios y fraternales (capítulo VI La Mente Patriarcal) "Así como domina el pater-familias sobre "su" mujer y "sus" hijos, domina en nosotros la voz de la sociedad patriarcal represiva sobre la voz de nuestro aspecto materno y sus valores matrísticos, e igualmente sobre nuestro "niño interior".

En su libro Transformar la educación para cambiar el mundo, Claudio Naranjo afirma que "es posible operar una trasnformación, una sanación que libere nuestra realidad interior y nos permita tener relaciones sanas con nosotros mismos, con los demás y con el entorno" Con esa esperanza abordamos nuestro trabajo como educadores.

Los valores patriarcales están cristalizados dentro de nosotros incluso cuando nuestro pensamiento racional los rechaza. Por eso encontramos una gran dicotomía entre el lenguaje de la ética, que representa lo que pensamos que es bueno y el lenguaje del deseo que genera la atracción y mueve las acciones.

Varias líneas de investigación estudian cómo las interacciones sociales conforman los modelos de atractivo. El estudio de Gómez (2004), J. demuestra "la existencia de una socialización mayoritaria, no única ni exclusiva, que fomenta que los modelos más atractivos sean aquellos ligados real o potencialmente a la violencia y la conflictividad.“ 
A través de dicho proceso de socialización, se crea una brecha entre lo bueno y lo deseable, dirigiendo el atractivo hacia relaciones afectivo-sexuales basadas en estructuras desiguales y de poder, generando atractivo hacia las personas que no tratan bien (Flecha; 2012; Gómez, 2004; Valls, Puigvert \& Duque, 2008).

Para acabar con esta situación de incoherencia entre lo que todos deseamos, y está descrito en cualquier decálogo por la paz, y lo que en realidad hacemos en el día a día aunque nos haga sufrir, las personas con responsabilidad educativa, necesitamos enfocar urgentemente nuestros esfuerzos en ayudar a nuestro alumnado a tomar conciencia de ese desajuste y que esa conciencia le lleve a planificar con determinación su compromiso de cambio hacia un nuevo modelo de relaciones que les de mayor felicidad.

La propuesta que presentamos está basada en una dinámica de personajes simbólicos que representan aspectos de una realidad interior difícil de aceptar. Hemos recurrido a la estrategia del juego por su gran valor terapéutico y por la distancia simbólica que genera, poniendo a salvo el yo.

Winicott decía que el juego es "una zona intermedia entre la realidad interna del individuo y la realidad compartida del mundo, que es exterior a él" El juego se desarrolla en ese espacio de descanso donde está permitido expresar todos los deseos y así poder aceptar las limitaciones y normas del entorno.

Vigotski decía que el niño-a cuando juega está un poco por encima de sí mismo, dado que jugando aumenta su zona de desarrollo próximo.

En las Comunidades de Aprendizaje planteamos la creación de contextos en los que se desarrolle el diálogo igualitario y el trabajo interactivo que potencia las relaciones de solidaridad, favoreciendo la eliminación de cualquier abuso y el desarrollo de un nuevo modelo de masculinidad respetuoso. El modelo dialógico de resolución de conflictos o modelo comunitario "implica que toda la comunidad participe en un diálogo que permita descubrir las causas y orígenes de los conflictos para solucionarlos desde la propia comunidad mucho antes de que aparezcan" (Flecha R. \& Garcia, C. (2007)

Pero necesitamos aprender a dialogar de forma no violenta, logrando que las palabras sean ventanas no paredes, como lo expresa Ruth Berermeyer en un poema citado por Marshall B. Rosenberg en su libro "La comunicación no violenta, un lenguaje de vida", donde propone el desarrollo de habilidades para la comunicación respetuosa.

En las Escuelas Restaurativas se trabaja por un modelo de justicia que lleve a tomar conciencia de los propios actos, asumiendo la responsabilidad y la reparación de daños.

La propuesta que presentamos aquí bebe de todos estos enfoques.

\section{Desarrollo}

\section{1. ¿Quiénes somos? ¿Dónde surge esta propuesta?}

El CEIP Huerta de Sta. Marina es un colegio público de Educación Infantil y Primaria, con 477 alumnas y alumnos de 3 a 12 años, situado en la zona norte del casco histórico de la ciudad de Sevilla, en el denominado Distrito Casco Antiguo.

Aunque el nivel cultural de la mayoría de las familias es medio- alto, la situación económica no se corresponde con ello en un gran porcentaje. El alumnado inmigrante representa un número significativo del total. Pero hemos de decir que la situación social de estas familias 
puede llegar a ser muy diferente, por lo tanto, no se les puede considerar como un grupo homogéneo.

Hay familias de procedencia germánica que valoran que sus hijos e hijas estén en una escuela pública que cuenta con un programa bilingüe de alemán. Otras familias inmigrantes llevan bastante tiempo instaladas en el barrio y tienen un nivel cultural medio o alto, en muchos casos son parejas mixtas (español/a y de otra nacionalidad). Otro grupo de familias las constituyen inmigrantes con situaciones económicas muy precarias dada la falta de empleo, su inestabilidad y las propias condiciones laborales tan pésimas. Son madres y padres que, caso de tener trabajo, tienen unas jornadas laborales interminables.

Hay un grupo muy numeroso de familias en las cuales uno o dos de los progenitores son docentes (infantil, primaria, secundaria, universidad).

En general podemos destacar que tienen un perfil sociológico democrático, con una alta vinculación en el tejido ciudadano y participativo del barrio y, por supuesto, con una gran sensibilización hacia los temas educativos y la vida escolar de sus hijos e hijas.

Desde septiembre de 2014 nuestro centro pertenece a la Red Andaluza de Comunidades de Aprendizaje, acogiéndonos a la normativa de la Junta de Andalucía, Orden de 8 de junio de 2012.

Nuestro proyecto educativo describe la escuela inclusiva con la que soñamos, la escuela de todos/as, donde la educación para "ser" está por encima de la educación para "tener" Una escuela donde se promueve la participación y el aprendizaje de todo el mundo. Una comunidad educativa donde se pretende que todos y todas nos sintamos acogidos y respetados por lo que somos, sin tener que demostrar ni hacer nada para merecerlo. A la vez una escuela donde se motiva y apoya con determinación el desarrollo y adquisición de talentos y cualidades diversos, fomentando altos niveles de logro para todo el alumnado, sin excluir a nadie.

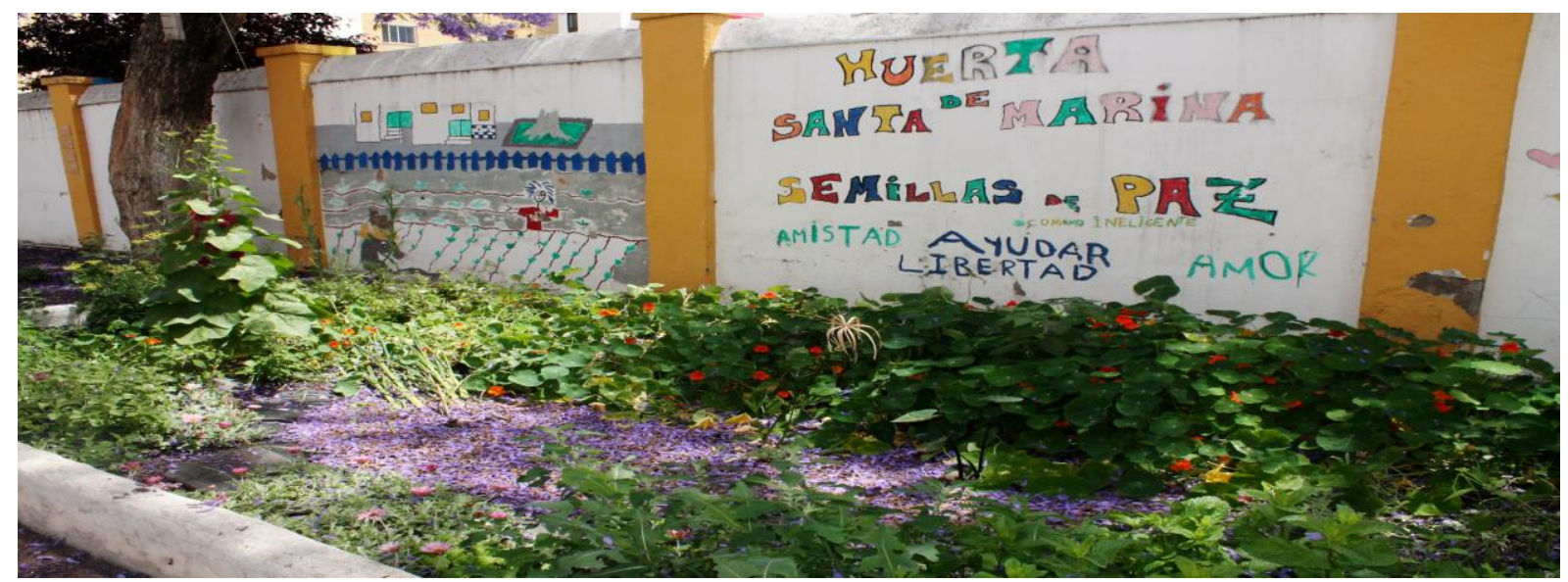

Cada año damos pasos a nivel organizativo y de formación para acercarnos a este sueño.

¿Y cómo es la convivencia en nuestra escuela?

Podemos decir que muy positiva, dado que en nuestro entorno la violencia explícita es una excepción. Son poco frecuentes los casos de agresiones físicas y verbales, aunque existen esporádicamente y nos gustaría que desapareciesen totalmente. Sin embargo nos preocupan mucho ciertos malestares que surgen de otro tipo de conflictos 
con una violencia más escondida y menos evidente y que tristemente se manifiesta en todos los grupos humanos: las dinámicas de exclusión por ser diferente, la manipulación de unos sobre otros, los juicios de valor, el afán desmesurado de protagonismo y/o de éxito, la competitividad tomando como referencia a los demás, la dificultad para perder o ceder, la falta de empatía, poner por delante del bien común los intereses personales, el empecinamiento o cerrazón para imponer la propia voluntad, la falta de respeto por lo colectivo .... dinámicas típicas de la sociedad patriarcal en la que vivimos.

En este terreno adultos y alumnado, todos, tenemos mucho que aprender para mejorar nuestras relaciones. Creemos que la escuela es un espacio ideal para ello, pero desde el enfoque del aprendizaje dialógico, donde la interacción con personas diferentes tiene un papel fundamental. Queremos ser una comunidad de personas donde las relaciones humanas mejoren día a día, enriqueciéndose con las diferencias de cada uno, aprendiendo a respetarlas y valorarlas. Con este propósito elegimos de forma participativa "el Respeto" como la Norma de convivencia de nuestra comunidad. Un concepto que queremos interiorizar profundamente porque es la base de la comunicación que nos hace felices.

En nuestro Plan de Acción Tutorial y en el Plan de Convivencia, hablamos de tres tipos de respeto: a nosotros mismos, a los demás y al entorno. En la planificación que realiza el profesorado, dedicamos cada mes del trimestre a profundizar en uno de los tres aspectos.

\section{2. ¿Cómo surgió la propuesta?}

\subsubsection{Fase 1: Gestación de la idea}

Al terminar el curso 2015/16 y realizar la valoración anual sobre el estado de la convivencia en nuestro centro, algunas personas de la comisión de convivencia decidimos analizar los conflictos más frecuentes que durante los 9 años anteriores se repetían entre el alumnado, observando que se podían reducir a 4 tipos de situaciones:

- Conflictos derivados de exclusiones y/o burlas: "No me dejan jugar, no me dejan participar, se burlan de mí, no me eligen ...

- Conflictos por actitudes violentas, con uso de la fuerza o la amenaza, sobre todo al perder en los juegos.

- Interrupciones frecuentes para conseguir atención

- Aceptación y/o participación en situaciones de exclusión por miedo a contradecir o enfrentarse a alguien.

Nos preguntamos sobre las dificultades que impedían que estas situaciones no se superasen y se repitieran cuando las personas implicadas habían tomado el propósito de conseguirlo. Planteamos varias preguntas:

- ¿qué hay detrás de una conducta irrespetuosa hacia los demás y hacia sí mismo? ¿qué quiere decir y qué consigue con esa forma de comportarse?

- ¿qué pensamientos y emociones la sostienen?

- ¿cómo ayudar a salir de ese círculo negativo que genera tanto malestar? 
Queríamos pedir la opinión del alumnado para poder contestar estas preguntas y para ello buscamos un personaje con un nombre para poder identificar cada tipo de actitud. Elegimos nombres inventados uniendo dos animales que nos sugerían dicha actitud.

\subsubsection{Fase 2: Definición de los personajes}

Presentamos los 4 personajes al alumnado de $6^{\circ}$ durante el curso 2016-17, diciéndoles que estábamos diseñando una dinámica para ayudar a todos los niños y niñas del colegio a prevenir y solucionar lo más rápido posible los problemas de convivencia que les surgían y que para ello necesitábamos su ayuda.

Preguntamos en primer lugar si los personajes les resultaban conocidos y si creían que faltaba alguno más.

De ese primer debate con el alumnado surgió la necesidad de definir, frente a las características de los personajes presentados, la del Ser Humano que no se deja manipular, que actúa con justicia y respeto. Elegimos el color verde para identificar lo que toda persona en esencia es: sabia y feliz.

Después comenzamos con el análisis de cada personaje, asignándole a cada uno un color, para poder identificarlo con una cartulina:

.- Leopave, color rosa; Lobogre, color rojo; Loromosca, color azul; cucarato, color amarillo

Queríamos descubrir: ¿qué ideas están detrás de su conducta, qué cree, por qué lo hace?, ¿qué sentimientos tiene?

Antes de hablar les pedimos que interpretaran con gestos y movimientos al personaje, que recordaran alguna situación real en la que habían actuado como ese personaje y si no tenían ninguna, que la imaginaran. En parejas compartieron las situaciones elegidas y extrajeron los pensamientos y sentimientos.

Después en gran grupo, con la participación de todos-as, fuimos elaborando la lista de pensamientos, descubriendo al contrastarlos con lo que piensa el sabio feliz, que todos ellos son equivocados, porque parten de un error fundamental y que ese error les lleva a actuar de una forma irrespetuosa que aunque pueda parecer que ganan algo, a la larga tiene consecuencias negativas y en absoluto consiguen lo que en el fondo buscan, no pueden estar en paz porque sus logros no les aporta verdadera felicidad .

Vimos que el error común a todos es la pérdida de la certeza del propio valor, la pérdida de la confianza básica en uno mismo.

De la confianza en el propio valor surge la sabiduría y la felicidad, pero muchas veces está dormida. ¿Por qué unas veces aflora fácilmente y otras no?

Concluimos que tristemente la gran mayoría de las personas perdemos esa confianza y por eso conocemos tan de cerca a estos personajes. Algunos nos manipulan con más frecuencia y más tiempo que otros. Podemos verlos en nosotros mismos, aunque eso cuesta un poco más, pero lo que resulta fácil es verlos en las personas que nos rodean. Lo más importante es darse cuenta cuanto antes, porque las actitudes de los personajes producen un efecto de contagio que es muy peligroso. 


\subsubsection{Fase 3: Elaboración de antídotos}

¿Cómo evitar los hechizos? Queríamos encontrar una manera rápida y eficaz de despertar al sabio escondido detrás de un personaje cuando lo identificamos, ya sea en nosotros mismos o en los demás.

Del debate surgió que podríamos encontrar una frase y un gesto que presentara de forma sintética la información correcta, que resultase como un antídoto frente al error. De esa forma se creaba una señal que podría evitar continuar en el camino equivocado y facilitar el rescate hacia una respuesta más sabia.

De todas las propuestas que surgieron se votó para decidir la que más nos gustaba.

Con todas las aportaciones definimos el perfil de cada personaje, concretando el pensamiento clave de su error y el antídoto que lo contrarrestara, que le permitiera a la persona que lo representa darse cuenta, para salir del personaje y volver a su Ser.

Para poder difundir esta información de una manera asequible a todos los niños y niñas del centro y que fácilmente pudiera ser memorizada para poder ponerla en práctica, buscamos posibles juegos.

Quedaron varias posibilidades en proyecto: un juego de mesa tipo "la oca", un juego de ordenador, un juego de representación de escenas... Pero el formato que hemos llevado a cabo hasta ahora con éxito es el de "pilla pilla congelador".

También buscamos una imagen para representarlos. Se propuso a todo el alumnado de $5^{\circ}$ y $6^{\circ}$ un concurso de dibujo. Entre todos los presentados eligieron por votación la imagen que querían.

\subsection{Objetivos que se pretenden con esta dinámica.}

1.- Aprender a identificar en uno mismo y en los demás, las conductas que generan conflictos, visualizando el pensamiento erróneo que está detrás y el sentimiento que provoca ese pensamiento.

2.- Concentrar toda la intención en evitar que el personaje te domine, buscando la salida más sabia a cada situación

3.- Crear una separación simbólica entre la persona y su conducta, entre el Ser y el Hacer, con el objeto de que toda la responsabilidad y esfuerzo del Ser se enfoque en controlar el Hacer. Es decir, garantizar la seguridad afectiva y el valor de toda persona incluso cuando se equivoca, porque si restaura esa confianza básica encontrará la fuerza para cambiar sus conductas. Tratar las conductas equivocadas desde la inclusión. La conducta inaceptable debe ser rechazada pero no la persona. Es la propia persona la que debe responsabilizarse para hacer el esfuerzo de acabar con su conducta violenta. "Tú sí, eso no" como propone Ángels García Cunyat 
4.- Evitar que la identidad de la persona quede etiquetada, atrapada en el rol de víctima o de agresor y crea que no tiene posibilidad de salir.

5.- Conocer los antídotos, es decir las ideas o pensamientos que pueden ayudar a salir de un conflicto. Y dar los pasos adecuados para hacerlo.

6.- Cuando el conflicto ya ha sucedido, facilitar la toma de conciencia de los errores agilizando el proceso de resolución y reparación.

\subsection{Descripción y presentación de los personajes}

Para dar a conocer esta dinámica entre todo el alumnado del centro estamos utilizando 5 tarjetas de colores.

Una verde que representa nuestra esencia, algo que ya está dentro de cada persona, que se puede conocer o permanecer oculto a uno mismo. Sobre esa tarjeta verde, a veces se sobrepone algún hechizo, representado por 4 tarjetas: azul, roja, rosa o amarilla. La parte sabia entonces queda tapada, oculta, pero está ahí, no desaparece. Cuando el antídoto funciona, el hechizo cae y el verde vuelve a estar en primer plano.

\subsubsection{Características de la persona sabia.}

- Confía en su valor como persona, un valor que no se puede perder y que es el mismo en todos los seres humanos, ni más ni menos.

- Sabe que tiene potencialidades y talentos únicos que puede desarrollar, pero su valor como persona no depende de ello.

- Tiene curiosidad por conocer los talentos diferentes de otras personas, porque sabe que colaborando se multiplica la fuerza y la riqueza.

- Sabe que hay muchísimas cosas que no sabe. Por eso tiene tantas ganas de aprender

- No tiene miedo a equivocarse, no le da vergüenza cuando le pasa, porque sabe que tiene muchas limitaciones, que no es perfecta, que equivocarse es inevitable y una buena forma de superarse.

- Sabe pedir ayuda cuando la necesita y la agradece.

- Expresa claramente sus necesidades y sentimientos y se asegura de que le han escuchado. Busca ayuda cuando no lo consigue.

- Escucha a los demás para ponerse de acuerdo. Le interesa el valor de los argumentos.

- No tiene miedo a perder o a ceder, sabe que su valor no depende en absoluto de tener la razón o del éxito.

- Respeta los acuerdos. Tiene paciencia para esperar su turno. Busca la justicia cuando no se respeta - Confía en que "hay suficiente para todos-as" (sitio, recursos, oportunidades...) si se reparte con justicia. Y defiende el mismo derecho para todos-as.

- Le importa y se siente mejor cuando las personas, los seres vivos y todo lo que le rodea es respetado, porque sabe que está unido a ellos. Se ocupa de ayudar para que así sea, dentro de sus posibilidades. 


\subsubsection{Los personajes que nos hechizan.}

Todos somos sabi@s, pero en ocasiones aparecen unos personajes que consiguen que olvidemos todo lo que sabemos y nos dominan. Entonces nos comportamos de una forma totalmente absurda que nos amarga la vida y se la amargamos a los que nos rodean.... nos han hechizado. A veces conseguimos despertar muy rápido, otras los personajes se quedan tanto tiempo que casi te crees que eres así. Hemos identificado a cuatro: a) lobogre, b) cucarate, c) leopave y e) loromosca.

\section{LEOPAVE (tú sí, tú no). Ilustración : Lorca Gutiérrez Prada}

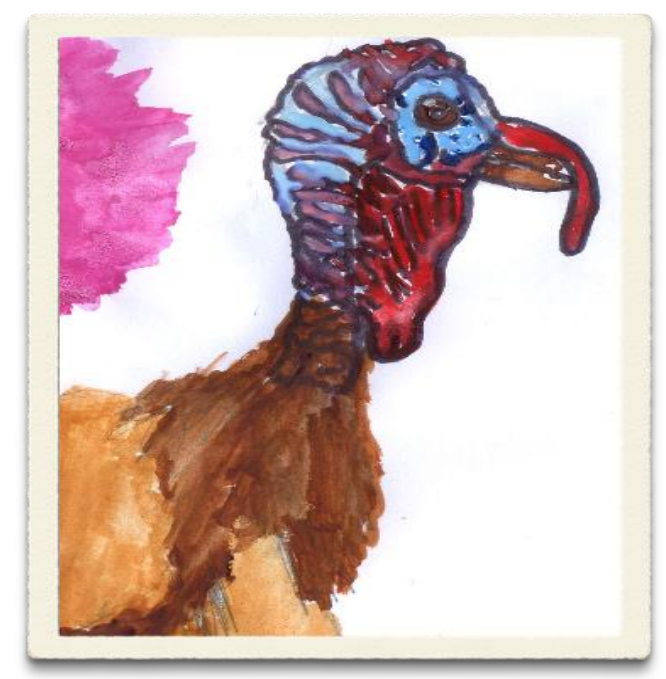

que yo diga.

\section{Errores en los que cree}

Algunas personas tienen más valor que otras. El valor depende del éxito, de lo que tienen, de lo que saben, de lo que hacen, de sus capacidades y talentos, ...

Es importante estar con los que valen más y dejar fuera a los que no, porque "no hay suficiente para todos". Es necesario organizar la situación para quedarse con lo mejor.

Tengo que ser "el/la mejor" el/la más "guay" para que los demás me hagan caso, para que hagan lo

$\square$ Me da vergüenza y miedo equivocarme, creo que así pierdo valor

$\square \quad$ Me alegro de los fracasos de los demás porque así tengo más posibilidades de éxito.

Sentimientos: Soberbia, orgullo, afán de control

Antídoto: ¡Adiós, hay sitio para todos!



DOI: http://dx.doi.org/10.12795/CP.2018.i27.09

\section{LOROMOSCA (Ilustración : Lorca Gutiérrez Prada)}

\section{Errores en los que cree}

Dudo de mi valor y necesito desesperadamente que me hagan caso para confirmar que soy importante. Si no me hacen caso creo que es porque no valgo.

Hago cualquier cosa para que se fijen en mí, insisto e insisto para que me vean.

No puedo esperar... tengo una enorme impaciencia porque temo quedarme sin atención. 
Sentimiento: Impaciencia

Antídoto: ¡Para! Ya eres importante



Errores en los que cree

Mi valor depende del éxito, de que consiga lo que quiero

Tengo que ganar y tener la razón por encima de todo. Si no me dan lo que quiero por las buenas lo consigo por las malas. La fuerza es mi forma de ganar.

Me da vergüenza equivocarme y me enfada mucho que se puedan reír de mí.

Me desahogo burlándome de las debilidades o equivocaciones de los demás. Así me consuelo creyéndome mejor que ellos-as

Sentimientos: Ira, enfado, resentimiento

Antídoto: "Espera!!! Puedes conseguirlo de otra manera!

\section{CUCARATE (Ilustración : Lorca Gutiérrez Prada)}

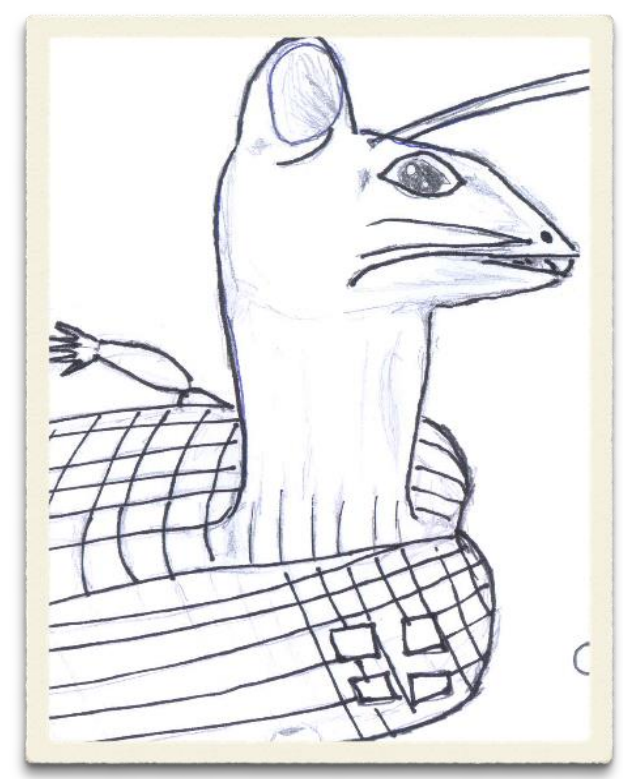

\section{Errores en los que cree}

* Dudo de mi valor, no me atrevo a expresar mis ideas, prefiero aceptar o copiar las de los que valen.

*Hago y aguanto lo que sea para que me acepten. Aunque me duela el abuso es mejor que quedarme solo-a. Escondo mi malestar.

*Temo expresar lo que pienso y cómo me siento por miedo a las represalias

Sentimientos: Miedo, culpa, frustración, desesperanza

Antídoto: "Exprésate!!! Tú vales!

\subsection{Cómo lo estamos trabajando con el alumnado.}

\subsubsection{Premisa básica}

Tras la reflexión de que el error común subyacente a todos los conflictos que observamos es la pérdida de la certeza en el propio valor es decir, la fragilidad de la confianza básica en uno mismo, decidimos estructurar nuestro Plan de Acción Tutorial 
teniendo muy en cuenta el respeto a uno mismo. Pero ¿qué es respetarnos a nosotros mismos? Cuanto más reflexionamos sobre ello, más matices encontramos. Nos parece fundamental señalar las diferencias entre Ser, Tener y Hacer.

El Ser de cada persona es único y valioso. Un valor que no podemos perder ni ganar y que está en todas las personas igual.

Sin embargo cada persona tiene unos talentos, potencialidades, cualidades, capacidades, diferentes que se pueden desarrollar o adquirir en mayor o menor medida.

Es decir, cada ser tiene su propia gama de colores original. Sacarlos todos a la luz para que brillen es el objetivo de la educación. Pero para que eso pueda suceder es fundamental garantizar en primer lugar el respeto al valor indiscutible de cada uno.

Cuando dudamos de nuestra valía, cuando creemos que nuestro color no es adecuado, nos hacemos daño y perdemos la fuerza necesaria para afrontar y superar cualquier dificultad.

Aprender despacio o deprisa no tiene nada que ver con el valor, cada persona tiene su ritmo y sus propias capacidades, por eso nunca debería darnos vergüenza cuando fallamos en algo o cuando otros consiguen ir más rápido. Lo importante es superarnos, sacarle todo el brillo posible a nuestros tonos. La comparación con los demás, creyéndonos mejores o peores es absurda y nos daña.

\subsubsection{El juego "No dejes que te hechicen, eres sabi@”}

En las clases de Educación Física se propone con cierta frecuencia con el formato pilla-pilla este juego, para memorizar e identificar de una forma lúdica lo esencial de cada personaje:

Un grupo de sabios es perseguido por 4 ó 5 Leopaves. Cuando te rozan con la mano te congelan dejándote inmovilizado en la postura de un leopave, (muy chulito y con el dedo índice señalando: tú sí, tú no). Te salvas si algún sabio consigue acercarse y decir el antídoto con el gesto acordado: "Adiós, hay suficiente para todos" girando una vuelta completa con los brazos abiertos.

En el siguiente turno los que pillan son los Lobogres, que llevan las manos como zarpas amenazantes y enseñando los dientes. El antídoto para deshechizar a los congelados es: "Espera, puedes conseguirlo de otra manera", con la mano izquierda abierta levantada y la derecha con el índice girando en círculos hacia delante.

Los Loromosca saltan continuamente llamando la atención con las manos levantadas. El antídoto es: “¡Para, ya eres importante! " Con las dos manos abiertas de frente y luego juntándolas y haciendo una inclinación de reverencia.

Los Cucarate van encorvados, tapándose la boca. El antídoto es decirles: "Exprésate, tú vales", abrazándoles.

El juego se va complicando progresivamente poniendo como perseguidores a más de un tipo de personaje.

\subsubsection{Otras actividades}

Cada tutor o tutora en el horario reservado para la acción tutorial con su grupo o en las sesiones de grupos interactivos plantea diferentes actividades en función de la edad de su alumnado, por ejemplo: 
- Análisis de los diferentes personajes que están descritos en las primeras páginas de la agenda escolar o utilizando las tarjetas de colores.

- Compartir libremente en asamblea situaciones en las que recordamos haber sufrido algún hechizo y lo que hicimos

- Analizar los pasos a dar por una persona sabia al identificar a alguno de los personajes

- Dibujar y/o representar escenas con diferentes finales.

- Identificación de personajes: Presentación de escenas donde se describe un conflicto y deben identificar el personaje o personajes implicados, analizar sus errores, buscar una respuesta sabia y representarlo. Exponemos algunos ejemplos de situaciones propuestas:

- Situación 1.- En el gimnasio hay varias pelotas saltarinas. Voy a coger una y en ese momento llega una compañera y la agarra. Las dos empezamos a tirar y empujar hasta que ella se la lleva. Cuando está usándola voy por detrás y le empujo. (Hechizo tipo lobogre)

- Situación 2.- Felipe y varios compañeros-as van a jugar a baloncesto en el recreo. Están preparando los equipos y Marta se acerca para decir que quiere participar. Felipe mira a Juan poniendo cara de disgusto y diciéndole al oído que Marta- no sabe jugar bien, que le diga que no. Entonces Juan dice que los equipos están completos, que no puede jugar. . (Hechizo tipo leopave y cucarato)

- Situación 3.-Nos sentamos en círculo para una asamblea. Hay una compañera que no tiene sitio. Yo estoy al lado de mis dos amigas y me acerco más a ellas cerrando el hueco para que no se siente a mi lado. (Hechizo tipo leopave)

- Situación 4.- Estoy en el recreo con mi grupo de amigos, veo que se acerca un compañero que no me gusta. Les digo discretamente que nos vayamos corriendo a otra zona para que no esté con nosotros. (Hechizo tipo leopave y cucarato)

- Situación 5.-Estamos en la asamblea, yo quiero decir algo pero mi turno tarda mucho en llegar. Insisto levantándome y como no me hacen caso me pongo a hablar y a hacer ruidos. (Hechizo tipo loromosca)

- Situación 6.- Jugamos al pilla pilla. Lucía la queda y me persigue, cuando me pilla yo digo que no vale, que estoy cansado y he parado un momento para ir a beber. No quiero admitir que me ha alcanzado y no quiero quedarla. Ella insiste y otros compañeros le ayudan diciéndome que me toca quedarla. Yo me enfado y me voy corriendo sin hacer caso. (Hechizo tipo lobogre)

En círculos de paz, con las personas implicadas en un conflicto concreto al analizar qué ha pasado, la identificación de los personajes que han participado ayuda a determinar con más rapidez y eficacia la responsabilidad de cada uno, plantear la reparación necesaria y establecer un compromiso de qué se necesita cambiar en el futuro. Transcribimos un ejemplo de diálogo:

Un grupo de niños y niñas de 6 años están jugando al pilla pilla. De pronto José, que es de los que son perseguidos se lanza sobre Daniel que es de los que pillan, le agarra por los hombros empujándole. Daniel se queda en el suelo llorando. La profesora le dice a José que se siente en el banquillo.

Más tarde en un círculo de paz los dos niños implicados y cuatro que estaban cerca cuando pasó analizan con una profesora la situación: 
- Daniel dice: me ha dolido mucho cuando me has agarrado y empujado, no quiero que vuelvas a hacerlo más. Así no me gusta jugar contigo.

-¿Qué hechizo has tenido José?:

- El de lobogre, quería que Daniel me persiguiera a mí, que me hiciera caso como a los demás, porque perseguía a todos menos a mí, entonces le agarré

-¿Qué antídoto necesitabas José para que el lobogre no te hechizara?

- "Espera! Puedes conseguirlo de otra manera!

- ¿De qué otra manera podías expresar lo que querías?

- Con palabras, diciéndole a Daniel que no soy invisible, que yo también juego. Se lo dije una vez pero no me hacía caso...

- ¿Si no te hacen caso qué hace un sabio en vez de pegar?

- Puede esperar y decirlo de nuevo

- Y si te siguen sin hacer caso podría pedir ayuda a la profe,

- o esperar a que acabe la partida y decirlo para que no se repita...

- Ahora vamos a representar la escena otra vez sin lobogre, y le dices a Daniel cómo te sientes

- Daniel escúchame, yo también juego, me siento como si fuera invisible, quiero que me persigas a mí también por favor, !!

- Vale José, pero no me gusta que me obligues a hacerlo y menos que me empujes

- Siento haberte hecho daño, estoy aprendiendo a esperar y a usar las palabras.

- De acuerdo, no eres invisible para mí.

\section{CONCLUSIONES}

1. Con el desarrollo de esta dinámica estamos comprobando que tomar conciencia del pensamiento equivocado que está detrás de las conductas irrespetuosas ayuda progresivamente a desactivarlas, encontrando más fácilmente respuestas alternativas y por tanto un mayor margen de libertad en las acciones.

2. Observamos que la utilización de personajes facilita mucho el reconocimiento de los propios errores, gracias a la distancia simbólica que se crea entre la persona y su conducta. Como consecuencia aumenta el deseo y el compromiso para cambiar.

3. Los personajes son una máscara que se puede poner y quitar, con ello se logra salir del encasillamiento o identificación de la persona con el rol de agresor o víctima.

4. Los 4 personajes, que representan cada uno de ellos un tipo de conflicto, coinciden en un mismo pensamiento erróneo: dudar del propio valor. Sin embargo la conducta que desarrolla para defenderse del malestar que ello les genera es diferente en cada uno, aunque en todos conlleva insatisfacción y problemas cada vez mayores.

5. El juego "no dejes que te hechicen, eres sabi@“" es una manera motivadora y efectiva de memorizar la información básica para la identificación y solución de errores propios y ajenos 
6. Creemos fundamental y prioritario para la acción educativa preservar y fortalecer la confianza básica de cada persona en su propio valor, es decir, lograr una buena autoestima, para conseguir interacciones sociales donde la comunicación sea verdaderamente respetuosa.

\section{Referencias bibliográficas}

Flecha, A.; Melgar, P.; Oliver, e. \& Pulido, C. (2010). Socialización preventiva en las Comunidades de Aprendizaje. Revista Inter universitaria de Formación del Profesorado $n^{\circ} 67(24,1) 89-100$.

Flecha, R., \& Garcia, C. (2007). Prevención de conflictos en las comunidades de aprendizaje. Idea La Mancha: Revista De Educación De Castilla-La Mancha, 4, 72-76.

García, A. (2018) Afrontar el acoso y otras conductas contrarias a la convivencia desde una perspectiva restaurativa. Revista Convives n²1, marzo de 2018, 5-11.

Gómez, J. (2004) El amor en la sociedad del riesgo: una tentativa educativa. Barcelona: El Roure.

Naranjo, C. (2010) La mente patriarcal. Barcelona: RBA Libros.

Naranjo, C. (2013) Cambiar la educación para cambiar el mundo. Barcelona: La llave.

Oliver, E., \& Valls, R. (2004). Violencia de género. Investigaciones sobre quiénes, por qué y cómo superarla. Barcelona: Hipatia.

Rosenberg, M. (2006) Comunicación no violenta: un lenguaje de vida. Buenos Aires: Gran aldea Editores.

Winnicott, D.W- (1971) Realidad y juego. Ed. Gedisa. Barcelona. 